\title{
SITUACIÓN EPIDEMIOLÓGICA DE LA INTOXICACIÓN POR SUSTANCIAS QUÍ́MICAS EN EL DEPARTAMENTO DEL META- COLOMBIA, PERIODO 2009-2014
}

\author{
Oscar Alexander Gutiérrez Lesmes ${ }^{1}$ \\ Nelly Johana Loboa Rodríguez ${ }^{2}$ \\ Laura Inés Plata Casas ${ }^{3}$
}

\section{RESUMEN}

Introducción: La intoxicación por sustancias químicas es un problema de salud pública, que afecta a la población de los municipios del departamento del Meta. Objetivo: Describir la situación epidemiológica de la intoxicación por sustancias químicas en el Meta. Metodología: Se realizó un estudio descriptivo de corte transversal, utilizando base de datos estatales de salud pública (2009-2014) constituida por 5.208 casos de intoxicación reportadas en este periodo, se calcularon la incidencia de periodo y la tasa de mortalidad; se analizaron variables epidemiológicas de morbilidad, mortalidad, tipo de sustancia, ubicación geográfica, tipo y vía de exposición, edad y sexo. Resultados: Los plaguicidas ocasionan el mayor número de intoxicaciones del Meta, causando el $68 \%$ de la incidencia y el $66 \%$ de la mortalidad, otro tipo de sustancias causan el $27,7 \%$ de los casos de morbilidad y $34 \%$ de la mortalidad. El 52,1\% de las intoxicaciones ocurren de manera accidental, seguidas por la intención suicida y ocupacional con el $21,2 \%$ y $18,1 \%$ respectivamente; las vías de exposición fueron la oral, la respiratoria y la dérmica. Discusión: Meta es un departamento caracterizado por alta producción agrícola y una frontera agrícola en expansión, lo cual explicaría la elevada incidencia de los plaguicidas, la utilización de sustancias químicas en suicidios ocurren debido al fácil acceso que se tiene de las sustancias, la exposición ocupacional está ligada a la actividad agrícola y pecuaria, la exposición accidental ocurre por manejo inadecuado de sustancias químicas en las viviendas y la contaminación ambiental. Conclusiones: la actividad agropecuaria del Meta, junto con actividades cotidianas como la limpieza y el control de plagas en las viviendas, traen consigo la utilización de sustancias químicas, esta proximidad permite la exposición a estas sustancias y la posterior intoxicación que generan morbilidad y mortalidad humana.

Palabras clave: intoxicación, riesgos ambientales, sustancias químicas, plaguicidas, hidrocarburos.

\section{EPIDEMIOLOGICAL SITUATION OF POISSONING WITH CHEMICAL SUBSTANCES IN THE DEPARTMENT OF META- COLOMBIA, PERIOD 2009-2014}

\section{ABSTRACT}

Introduction: Chemical poisoning is a public health problem, affecting the population of the municipalities of Meta. Objective: To describe the epidemiological situation of chemical poisoning in the department of Meta.

\footnotetext{
${ }^{1}$ Escuela de Salud Pública, Facultad Ciencias de la Salud, Universidad de los Llanos. Villavicencio, Colombia. E-mail: oagutierrez@unillanos.edu.co ORCID: 0000-0002-5181-0236

2 Maestría en Epidemiología, Facultad Ciencias de la Salud, Universidad de los Llanos. Villavicencio, Colombia. E-mail: johannaloboa@unillanos.edu.co johannaloboa@unillanos.edu.co

${ }_{3}^{3}$ Centro de Estudios Epidemiológicos, Facultad Ciencias de la Salud, Universidad de los Llanos. Villavicencio, Colombia. E-mail: Iplata@unillanos.edu.co ORCID: 0000-0002-0375-8875
} 
Methodology: A descriptive cross-sectional study was conducted, using statal public health data bases (2009-2014) consisting of 5,208 cases of poisoning reported in this period. The incidence, morbidity, mortality, substance type, geographic location, type and route of exposure, age and sex were analyzed. Results: Pesticides cause the highest number of poisonings of the Meta, causing $68 \%$ of incidence and $66 \%$ of mortality, and other types of substances cause $27.7 \%$ of cases of morbidity and $34 \%$ of mortality. $52.1 \%$ of the poisonings occur accidentally, followed by suicidal and occupational intent with $21.2 \%$ and $18.1 \%$ respectively; the routes of exposure were oral, respiratory and dermal. Discussion: Meta is a department characterized by high agricultural production and agricultural

\section{INTRODUCCIÓN}

La salud de la población está directamente relacionada con necesidad básica humana de un ambiente seguro, que se expresa en agua, aire, alimentos y vivienda adecuados $(1,2)$; la multicausalidad necesaria o suficiente para que un evento en salud ocurra, depende de condiciones ambientales (en este caso las sustancias químicas), económicas y sociales, condiciones presentes en el departamento del Meta (3).

Resulta indispensable para garantizar el derecho a la vida y al bienestar de los habitantes del departamento, determinar la degradación en la salud ambiental ocasionada por las sustancias químicas ligadas a la actividad humana.

Disponer de una descripción del estado actual de la situación epidemiológica de la intoxicación por sustancias químicas (plaguicidas, metales pesados, solventes, otras sustancias) en los municipios del Meta, permite reconocer los problemas de salud derivados de las sustancias químicas utilizadas en estos municipios, clasificarlo y visibilizarlo para que sea objeto frontier expansion, which would explain the high incidence of pesticides, the use of chemicals in suicides occur due to the easy access of substances; occupational exposure is linked to agricultural and livestock activities, accidental exposure occurs through improper handling of chemicals in homes and environmental pollution. Conclusions: Agricultural activity in the department of Meta, along with everyday activities such as cleaning and pest control in homes bring the use of chemicals, this proximity allows exposure to these substances and the subsequent intoxication which generates morbidity and human mortality.

Key words: intoxication, environmental risks, chemicals, pesticides, hydrocarbons.

en las intervenciones de salud pública. Esta descripción e identificación del comportamiento entre los municipios, posibilita desarrollar acciones que mitiguen el impacto antrópico en el ambiente y el efecto que el ambiente contaminado pueda tener sobre los grupos humanos.

\section{METODOLOGÍA}

Se realizó un estudio descriptivo de corte transversal, se utilizaron los 5.208 casos registrados en bases de datos estatales de salud pública de los municipios del Meta de los años 2009, 2010, 2011, 2012, 2013 y 2014, las cuales contienen atenciones médicas realizadas en los diferentes centros de salud, clínicas y hospitales de la red de instituciones prestadoras de salud. Las bases de datos fueron ajustadas, validadas y procesadas en el software estadístico SPSS versión 23 , el análisis se realizó mediante estadística descriptiva, los resultados son presentados en tablas y figuras.

El comportamiento de la incidencia y mortalidad en la línea de tiempo 2009 a 2014 no presentó diferencias estadísticas significativas entre años, 
este artículo presenta la situación epidemiológica acumulada en el periodo y bajo esta acumulación presenta los resultados.

\section{RESULTADOS}

En la tabla 1 se observa la edad de la población afectada por intoxicación según las sustancias, la proporción de menores de edad afectada por las intoxicaciones varía según cada sustancia química: en las intoxicaciones por plaguicidas el $30 \%$ son menores de edad, en las intoxicaciones por otras sustancias químicas son el $50 \%$ y por solventes el $70 \%$; el promedio de edad es diferente entre los grupos, siendo los intoxicados por solventes quienes tienen un promedio de edad más bajo (14,6 años).

Tabla 1. Edad en años de los casos según tipo de intoxicación en el Meta. 2009-2014

\begin{tabular}{lcccc}
\hline & Intoxicación por & $\begin{array}{c}\text { Itras sustancias } \\
\text { químicas }\end{array}$ & $\begin{array}{c}\text { Intoxicación por } \\
\text { plaguicidas }\end{array}$ & $\begin{array}{c}\text { Intoxicación } \\
\text { por solventes }\end{array}$ \\
\hline Media & 21,54 & 27,60 & 14,64 \\
Mediana & 18,00 & 24,00 & 7,50 \\
Moda & 1 & 16 & 1 \\
Desv. Est. & & 18,196 & 15,914 & 17,428 \\
& 10 & 1,00 & 13,00 & 1,00 \\
& 20 & 2,00 & 16,00 & 1,00 \\
& 30 & 11,00 & 18,00 & 2,00 \\
Percentiles & 40 & 16,00 & 21,00 & 3,00 \\
& 50 & 18,00 & 24,00 & 7,50 \\
& 60 & 22,00 & 27,00 & 14,00 \\
& 70 & 28,00 & 31,00 & 16,10 \\
& 80 & 35,60 & 40,00 & \\
\hline
\end{tabular}

Fuente: bases de datos estatales de salud pública de los municipios del Meta.

En la tabla 2 se observa la distribución de las intoxicaciones según el sexo, el sexo masculino es el que presenta más casos de intoxicación con plaguicidas y solventes, en la intoxicación por otras sustancias químicas no hay diferencia según el sexo.

Tabla 2. Distribución de sexo según tipo de intoxicación en el Meta. 2009-2014

\begin{tabular}{lccc}
\hline & $\begin{array}{c}\text { Intoxicación por otras } \\
\text { sustancias químicas }\end{array}$ & $\begin{array}{c}\text { Intoxicación por } \\
\text { plaguicidas }\end{array}$ & $\begin{array}{c}\text { Intoxicación por } \\
\text { solventes }\end{array}$ \\
\hline Femenino & $\%$ & $\%$ & $\%$ \\
Masculino & 50 & 35,1 & 40,5 \\
Total & 50 & 64,9 & 59,5 \\
\hline
\end{tabular}

Fuente: bases de datos estatales de salud pública de los municipios del Meta 
En la tabla 3 se observa el área de ocurrencia de la exposición, en la cabecera municipal ocurren el $73,8 \%$ de los casos y otras sustancias químicas
$78,4 \%$ de los casos, mientras que en el área rural predomina la intoxicación por plaguicidas con el $35,6 \%$ de los casos.

Tabla 3. Distribución de área de ocurrencia según tipo de intoxicación en el Meta. 2009-2014

\begin{tabular}{lccc}
\hline & $\begin{array}{c}\text { Intoxicación por otras } \\
\text { sustancias químicas }\end{array}$ & $\begin{array}{c}\text { intoxicación por } \\
\text { plaguicidas }\end{array}$ & $\begin{array}{c}\text { intoxicación por } \\
\text { solventes }\end{array}$ \\
\hline Cabecera municipal & $\%$ & $\%$ & $\%$ \\
Centro poblado & 78,4 & 57,5 & 73,8 \\
Rural disperso & 7,2 & 6,9 & 7,1 \\
Total & 14,4 & 35,6 & 19,0 \\
\hline
\end{tabular}

Fuente: bases de datos estatales de salud pública de los municipios del Meta

En la tabla 4 se observa tasa de incidencia acumulada y la tasa de mortalidad por intoxicación (calculadas por cada 100.000 habitantes) con sustancias químicas (consolidado de los diferentes tipos de intoxicación), los municipios con la incidencia más alta son: Fuente de Oro, 2.143 casos; San Carlos de Guaroa, 1.616 casos; San Martín, 1.175 casos; Cabuyaro, 1.156; El Castillo, 1.086; San Juan de Arama, 1.007; Barranca de Upía, 977 ; Restrepo, 888; Castilla la Nueva, 884; Puerto Lleras, 877 casos.
Las tasas de mortalidad más altas según municipio en orden descendente se registraron así: Villavicencio 35 casos, Granada 11 casos, Fuente de Oro 10, La Uribe 10, Puerto Gaitán 10, Mesetas 9, Puerto Lleras 7, San Martín 6; Acacías, Puerto López y Vistahermosa, cada uno con 5 casos; en los municipios como Cabuyaro, Castilla la Nueva, Cubarral, El Calvario, Lejanías, Restrepo, San Juanito no se presentaron casos. El comportamiento en el resto de municipios puede observarse en la tabla 4. 
Tabla 4. Tasa de incidencia acumulada y tasa de mortalidad de intoxicaciones por sustancia químicas por municipios del Meta. 2009-2014 (por cada 100.000 habitantes)

\begin{tabular}{|c|c|c|c|c|}
\hline Municipio & Número de casos & $\begin{array}{c}\text { Tasa de incidencia } \\
\text { acumulada }\end{array}$ & $\begin{array}{l}\text { Número de } \\
\text { muertes }\end{array}$ & $\begin{array}{c}\text { Tasa de } \\
\text { mortalidad }\end{array}$ \\
\hline Acacías & 348 & 547 & 3 & 5 \\
\hline Barranca de Upía & 36 & 977 & 0 & 0 \\
\hline Cabuyaro & 45 & 1156 & 0 & 0 \\
\hline Castilla la nueva & 77 & 884 & 0 & 0 \\
\hline Cubarral & 20 & 350 & 0 & 0 \\
\hline Cumaral & 131 & 747 & 0 & 0 \\
\hline El Calvario & 13 & 576 & 0 & 0 \\
\hline El Castillo & 71 & 1086 & 0 & 0 \\
\hline El Dorado & 28 & 825 & 0 & 0 \\
\hline Fuente de Oro & 268 & 2143 & 1 & 10 \\
\hline Granada & 458 & 791 & 6 & 11 \\
\hline Guamal & 47 & 509 & 0 & 0 \\
\hline La Macarena & 54 & 182 & 1 & 2 \\
\hline La Uribe & 25 & 261 & 1 & 10 \\
\hline Lejanías & 119 & 755 & 0 & 0 \\
\hline Mapiripán & 13 & 116 & 0 & 0 \\
\hline Mesetas & 45 & 234 & 2 & 9 \\
\hline Puerto Concordia & 0 & 0 & 0 & 2 \\
\hline Puerto Gaitán & 35 & 347 & 1 & 10 \\
\hline Puerto Lleras & 279 & 877 & 2 & 7 \\
\hline Puerto López & 130 & 710 & 1 & 5 \\
\hline Puerto Rico & 39 & 368 & 0 & 2 \\
\hline Restrepo & 75 & 888 & 0 & 0 \\
\hline San Carlos de Guaroa & 145 & 1616 & 0 & 3 \\
\hline San Juan de Arama & 21 & 1007 & 0 & 2 \\
\hline San Juanito & 36 & 153 & 0 & 0 \\
\hline San Martín & 174 & 1175 & 1 & 6 \\
\hline Villavicencio & 2228 & 498 & 157 & 35 \\
\hline Vistahermosa & 19 & 81 & 1 & 5 \\
\hline
\end{tabular}

Fuente: bases de datos estatales de salud pública de los municipios del Meta

En la tabla número 5 se observa la tasa de incidencia acumulada y la tasa de mortalidad (2009-2014) de los municipios del Meta (calculadas por cada 100.000 habitantes) para cada una de las diferentes intoxicaciones químicas reportadas. La distribución en orden descendente de los cinco municipios más afectados por la incidencia en cada una de las intoxicaciones es la siguiente: Intoxicación por plaguicidas: Fuente de Oro, San Carlos de Guaroa, El Castillo, Puerto Lleras, El Dorado; Intoxicación por otras sustancias químicas:
Restrepo, San Martín, Cabuyaro, El Calvario, San Juan de Arama; Intoxicación por solventes: San Juanito, Restrepo, Cubarral, Castilla la Nueva, Cumaral; para la intoxicación por metales pesados solo se presentaron casos en San Martín y Villavicencio.

La distribución de la mortalidad en los municipios del Meta presentó el siguiente comportamiento, los primeros cinco municipios más afectados según la tasa de mortalidad reportada para cada una de las intoxicaciones 
es la siguiente: Mortalidad por intoxicación Fuente de Oro, Granada, San Martín, La con plaguicidas: Villavicencio, Granada, La Uribe. No se presentaron casos de mortalidad Uribe, Mesetas, Puerto Gaitán; Mortalidad por para intoxicación con metales pesados ni con intoxicación con otras sustancias: Villavicencio, solventes.

Tabla 5. Tasa de incidencia acumulada y tasa de mortalidad por intoxicación química según tipo de sustancia y según municipio del Meta. 2009-2014 (por cada 100.000 habitantes)

\begin{tabular}{|c|c|c|c|c|c|c|c|c|}
\hline & \multicolumn{2}{|c|}{$\begin{array}{l}\text { Intoxicación con } \\
\text { plaguicidas }\end{array}$} & \multicolumn{2}{|c|}{$\begin{array}{c}\text { Intoxicación con metales } \\
\text { pesados }\end{array}$} & \multicolumn{2}{|c|}{$\begin{array}{l}\text { Intoxicación con } \\
\text { solventes }\end{array}$} & \multicolumn{2}{|c|}{$\begin{array}{l}\text { Intoxicación con otras } \\
\text { sustancias químicas }\end{array}$} \\
\hline & Incidencia & Mortalidad & Incidencia & Mortalidad & Incidencia & Mortalidad & Incidencia & Mortalidad \\
\hline Acacías & 307 & 5 & 0 & 0 & 35 & 0 & 205 & 0 \\
\hline $\begin{array}{l}\text { Barranca de } \\
\text { Upía }\end{array}$ & 618 & 0 & 0 & 0 & 26 & 0 & 333 & 0 \\
\hline Cabuyaro & 690 & 0 & 0 & 0 & 34 & 0 & 432 & 0 \\
\hline $\begin{array}{l}\text { Castilla la } \\
\text { Nueva }\end{array}$ & 540 & 0 & 0 & 0 & 57 & 0 & 286 & 0 \\
\hline Cubarral & 229 & 0 & 0 & 0 & 42 & 0 & 79 & 0 \\
\hline Cumaral & 520 & 0 & 0 & 0 & 48 & 0 & 179 & 0 \\
\hline El Calvario & 171 & 0 & 0 & 0 & 29 & 0 & 376 & 0 \\
\hline El Castillo & 932 & 0 & 0 & 0 & 11 & 0 & 142 & 0 \\
\hline El Dorado & 654 & 0 & 0 & 0 & 12 & 0 & 159 & 0 \\
\hline Fuente de Oro & 1807 & 5 & 0 & 0 & 47 & 0 & 290 & 5 \\
\hline Granada & 560 & 7 & 0 & 0 & 25 & 0 & 206 & 4 \\
\hline Guamal & 280 & 0 & 0 & 0 & 37 & 0 & 192 & 0 \\
\hline La Macarena & 120 & 2 & 0 & 0 & 8 & 0 & 54 & 0 \\
\hline La Uribe & 215 & 7 & 0 & 0 & 3 & 0 & 43 & 3 \\
\hline Lejanías & 571 & 0 & 0 & 0 & 13 & 0 & 171 & 0 \\
\hline Mapiripán & 46 & 0 & 0 & 0 & 17 & 0 & 53 & 0 \\
\hline Mesetas & 129 & 7 & 0 & 0 & 8 & 0 & 97 & 2 \\
\hline $\begin{array}{l}\text { Puerto } \\
\text { Concordia }\end{array}$ & 0 & 2 & 0 & 0 & 0 & 0 & 0 & 0 \\
\hline Puerto Gaitán & 193 & 7 & 0 & 0 & 11 & 0 & 142 & 3 \\
\hline Puerto Lleras & 708 & 5 & 0 & 0 & 39 & 0 & 130 & 2 \\
\hline Puerto López & 558 & 5 & 0 & 0 & 15 & 0 & 137 & 0 \\
\hline Puerto Rico & 297 & 0 & 0 & 0 & 5 & 0 & 65 & 2 \\
\hline Restrepo & 362 & 0 & 0 & 0 & 83 & 0 & 444 & 0 \\
\hline San Carlos de & 1276 & 3 & 0 & 0 & 46 & 0 & 294 & 0 \\
\hline $\begin{array}{l}\text { San Juan de } \\
\text { Arama }\end{array}$ & 597 & 0 & 0 & 0 & 61 & 0 & 349 & 2 \\
\hline San Juanito & 99 & 0 & 0 & 0 & 49 & 0 & 5 & 0 \\
\hline San Martín & 685 & 2 & 4 & 0 & 48 & 0 & 438 & 4 \\
\hline Villavicencio & 260 & 19 & 1,2 & 0 & 34 & 0 & 203 & 16 \\
\hline Vistahermosa & 70 & 5 & 0 & 0 & 4 & 0 & 6 & 0 \\
\hline
\end{tabular}

Fuente: bases de datos estatales de salud pública de los municipios del Meta. 
En la figura 1 se observan los productos reportados en las intoxicaciones con plaguicidas, siendo el rodenticida (Campeón) el producto que presenta la proporción más alta en los casos de intoxicación en el Meta, seguido del pesticida Furadan; se debe evidenciar la intoxicación con diversos productos químicos de uso agrícola, los cuales sumados representan el mayor grupo de productos; ahora, según grupo químico son los carbamatos y los organofosforados los responsables de la mayoría de los casos de intoxicación en el Meta.

Figura 1. Productos reportados en la intoxicación por plaguicidas en el Meta. 2009-2014

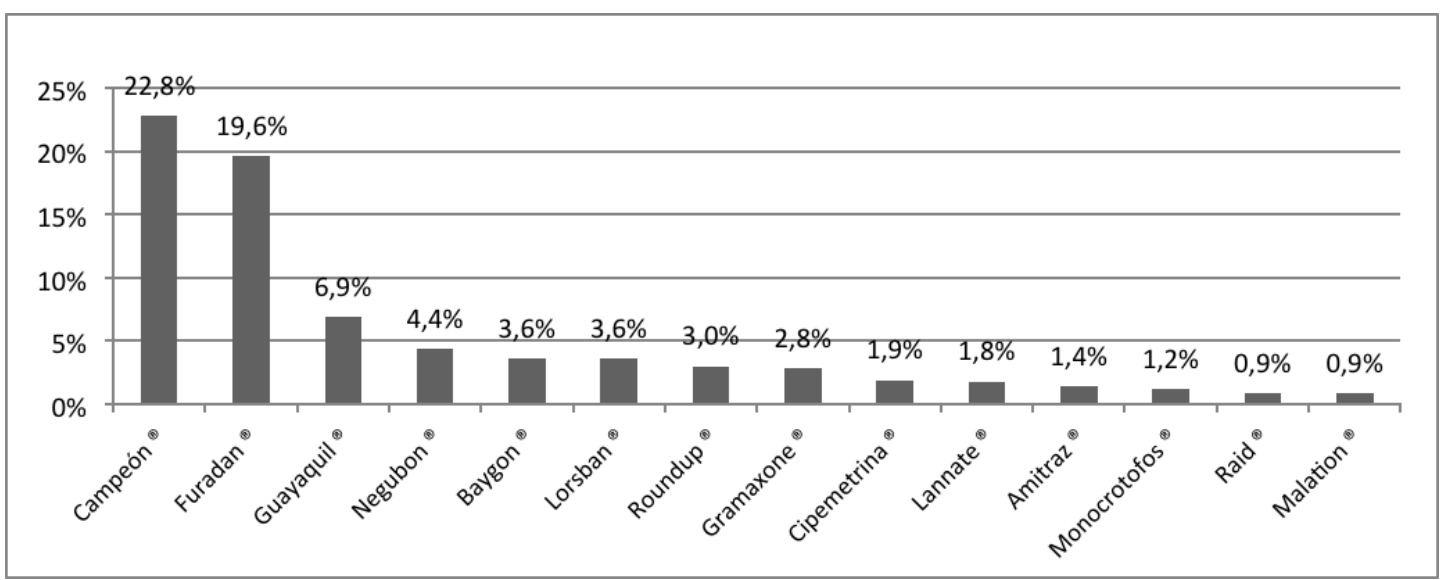

Fuente: bases de datos estatales de salud pública de los municipios del Meta

En la figura 2 se observan los productos reportados en las intoxicaciones por otras sustancias, siendo los utilizados en limpieza y desinfección los que acumulan más del $50 \%$ de los casos. Debe destacarse el cloro como la sustancia que más intoxicaciones reporta; la categoría otras sustancias de la figura agrupa 61 sustancias diferentes entre las que se encuentran productos de peluquería, productos de belleza, cuidado del cabello, cosméticos, cremas, cuidado del calzado, jabones, etc.

Figura 2. Productos reportados en la intoxicación por otras sustancia químicas en el Meta. 2009-2014

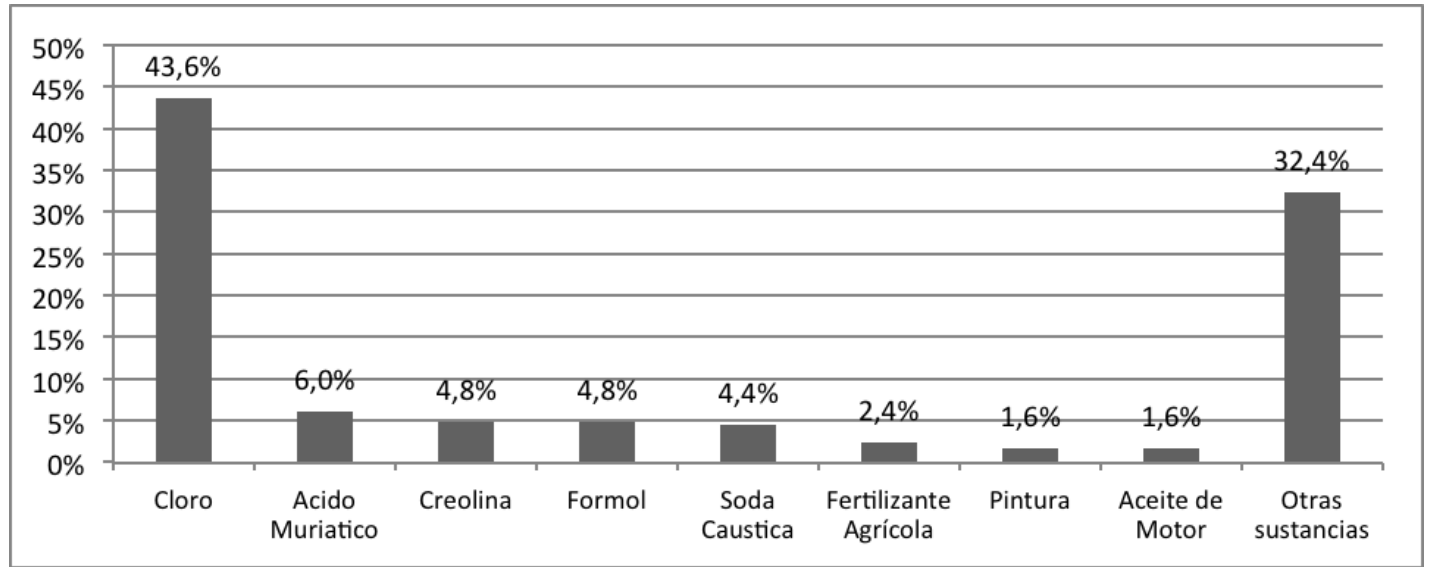

Fuente: bases de datos estatales de salud pública de los municipios del Meta 
En la figura 3 se observan los productos reportados en la intoxicación por solventes, siendo los productos usados para dilución de pinturas como thinner, varsol y acetona el grupo con mayor número de reportes, seguido de los combustibles como gasolina y aceite combustible para motores (ACPM).

Figura 3. Productos reportados en la intoxicación por solventes en el Meta. 2009-2014 (proporción)

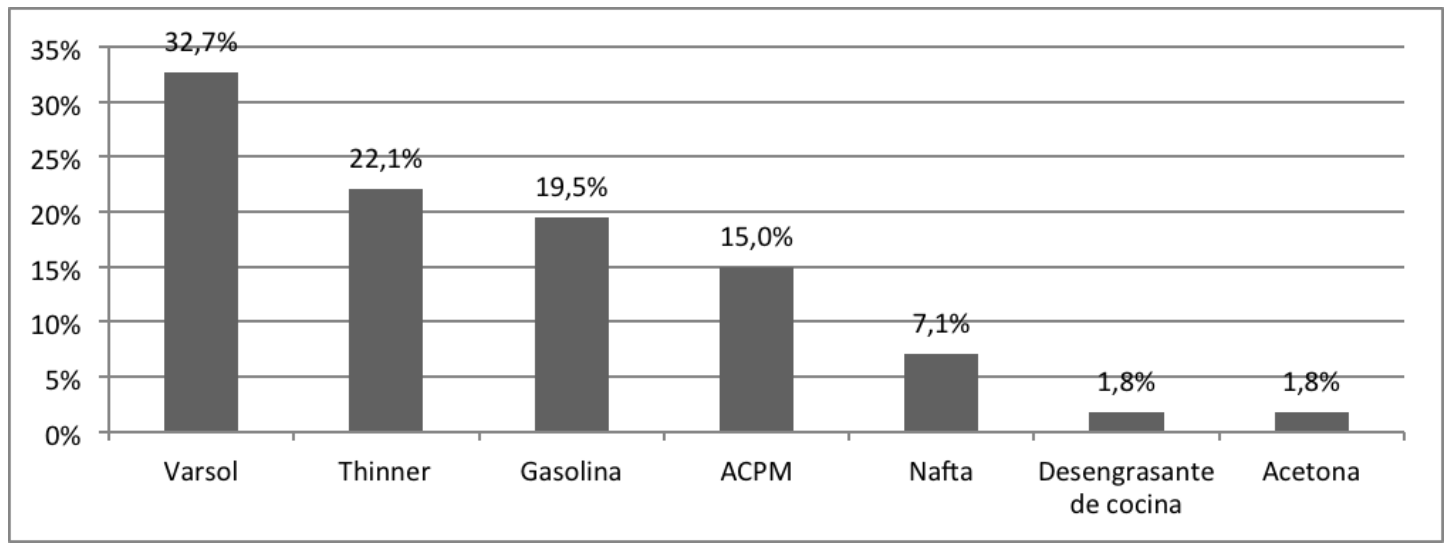

Fuente: bases de datos estatales de salud pública de los municipios del Meta.

En la tabla 6 se observa el tipo de exposición según la clase de sustancia, las intoxicaciones con plaguicidas ocurren en mayor proporción con fines suicidas, seguido por las intoxicaciones ocupacionales; las intoxicaciones con otras sustancias ocurren de manera accidental, seguidas de la intención suicida; la intoxicación con solventes ocurre principalmente por accidente, seguida de la intención suicida; la intoxicación por metales pesados ocurre de manera accidental y ocupacional.

Tabla 6. Tipo de exposición según tipo de intoxicación en el Meta. 2009-2014

\begin{tabular}{lcccccc}
\hline & Plaguicidas & $\begin{array}{c}\text { Otras } \\
\text { sustancias }\end{array}$ & Solventes & $\begin{array}{c}\text { Metales } \\
\text { pesados }\end{array}$ & \multicolumn{2}{c}{ Todas las intoxicaciones } \\
\hline & $\%$ & $\%$ & $\%$ & $\%$ & $\begin{array}{c}\text { Promedio } \\
\%\end{array}$ & IC 95 \% \\
Accidental & 23,3 & 47,2 & 63 & 75 & 52,1 & $33,2-69,0$ \\
Delictiva & 0,2 & 8,7 & 1,3 & 0 & 2,55 & $0,05-6,5$ \\
Desconocida & 1,2 & 9,5 & 7,9 & 0 & 4,65 & $0,3-8,7$ \\
Intencional homicida & 1,3 & 1,5 & 2 & 0 & 1,2 & $0,3-1,8$ \\
Intencional suicida & 44,9 & 24,7 & 15,2 & 0 & 21,2 & $6,1-37,4$ \\
Ocupacional & 28,6 & 8,3 & 10,6 & 25 & 18,12 & $9,4-26,8$ \\
\hline
\end{tabular}

Fuente: bases de datos estatales de salud pública de los municipios del Meta 
En la tabla 7 se observa la vía de exposición a la sustancia química con la cual ocurrió la intoxicación, en la exposición con plaguicidas se identificaron tres vías principales (oral, dérmica y respiratoria); en la intoxicación con solvente y otras sustancias se identificó el predominio de dos vías (oral y respiratoria); para las intoxicaciones con metales pesados el predominio fue ocular y respiratorio.

Tabla 7. Vía de exposición según tipo de intoxicación en el Meta. 2009-2014.

\begin{tabular}{|c|c|c|c|c|c|c|}
\hline & \multirow{2}{*}{$\begin{array}{c}\text { Plaguicidas } \\
\%\end{array}$} & \multirow{2}{*}{$\begin{array}{c}\text { Otras } \\
\text { sustancias } \\
\%\end{array}$} & \multirow{2}{*}{$\begin{array}{c}\text { Solventes } \\
\%\end{array}$} & \multirow{2}{*}{$\begin{array}{c}\text { Metales } \\
\text { pesados } \\
\%\end{array}$} & \multicolumn{2}{|c|}{ Todas las intoxicaciones } \\
\hline & & & & & Promedio & IC $95 \%$ \\
\hline Dérmica & 20,2 & 4,4 & 1,3 & 0 & 12,30 & $4,4-20,2$ \\
\hline Desconocida & 0,9 & 7 & 0 & 0 & 3,95 & $0,9-7$ \\
\hline Ocular & 1 & 0,9 & & 50 & ,95 & $0,9-1,0$ \\
\hline Oral & 58,6 & 71,7 & 82,1 & 0 & 65,15 & $58,6-71,7$ \\
\hline Parenteral & 0,1 & 0,2 & & 0 & 15 & $0,1-0,2$ \\
\hline Respiratoria & 18,4 & 15,4 & 15,2 & 50 & 16,90 & $15,4-18,4$ \\
\hline Transplacentaria & 0,3 & 0,4 & 1,3 & 0 & ,35 & $0,3-0,4$ \\
\hline
\end{tabular}

Fuente: bases de datos estatales de salud pública de los municipios del Meta

\section{DISCUSIÓN}

La exposición humana a sustancias químicas ocasiona efectos sobre la salud $(4,5)$ y dependiendo de la concentración de la sustancia en el ambiente, la vía y el tiempo de exposición, varía el nivel del impacto, sumado a esto la exposición varía de concentración en ambiente exterior o interior (6).

El desarrollo humano ha generado diversidad de productos químicos para el uso en actividades antrópicas, en Colombia se conocen entre 80.000 a 100.000 sustancias químicas de uso común, las cuales afectan la salud (7), diversas sustancias químicas circulan en los municipios de Meta, ocasionando morbimortalidad relacionada con ellas $(3,5)$. En los seis años analizados en la presente investigación, se registraron casos de intoxicación por plaguicidas, intoxicación por metales pesados, solventes y por otras sustancias, al igual que mortalidad por los mismos eventos.

El impacto de las sustancias químicas sobre la salud es reconocido por el Estado colombiano, por eso hace parte de las intervenciones del
Estado; en el CONPES3550 de 2008 (lineamientos para la formulación de la política integral de salud ambiental) se evidencia un énfasis sobre la seguridad química (8), también el Estado ha venido vigilando el efecto de las sustancias químicas sobre la salud humana mediante el sistema de vigilancia en salud pública (9).

El plan decenal de salud pública 2012-2021 contiene una dimensión de salud ambiental en la cual se aborda la problemática de las sustancias químicas como causantes de efectos sobre la salud (10). Esta dimensión busca promover la calidad de vida y la salud de la población mediante la trasformación de determinantes desde todos los sectores, uno de los objetivos es intervenir los determinantes sanitarios y ambientales de la salud relacionados con las sustancias y productos químicos, por esto plantea las siguientes metas relacionadas con las sustancias químicas: para el 2017 se deben tener priorizadas las entidades territoriales según problemas de salud ambiental relacionadas con sustancias químicas y la Política Nacional para la Gestión Integral de Sustancias Químicas que involucre todas las etapas del ciclo de vida de las mismas; para el 2021 el 100\% de las entidades 
territoriales de salud implementan la gestión integral de sus residuos peligrosos (10). En el plan decenal la gestión racional de los productos químicos en su ciclo de vida es fundamental para la protección de la salud humana. Este artículo permite observar el impacto de estas sustancias en los habitantes de los municipios del Meta, a través de su sistema de vigilancia en salud pública.

Sumado a lo anterior, existe en Colombia regulación sobre la producción, comercialización, uso y disposición final de las sustancia químicas según el grupo de sustancia químicas, el impacto y el sitio de uso. Lo anterior con la finalidad de reducir el impacto ambiental y el impacto sobre la salud humana.

A pesar de la existencia de normatividad en Colombia que regula la distribución, almacenamiento, comercialización, uso y disposición final de sustancias químicas, como por ejemplo en el uso de plaguicidas (decreto 1843 de 1991, decreto 1443 de 2014) con los cuales se regula el manejo adecuado de los plaguicidas y el no cumplimiento genera impacto sobre la salud (11), precisamente esto ha venido ocurriendo a los habitantes de los municipios del Meta, evidenciado por el impacto descrito (incidencia y mortalidad) en el artículo. El no cumplimiento de la normatividad puede obedecer a desconocimiento de la afección en salud a la que se expone la población (12) y los usuarios, a la falta de recursos por parte de los usuarios para contar con equipos de protección adecuados (13). En 2009 el Estado colombiano ya asumía que el 67,5\% de la enfermedades profesionales eran atribuidas a sustancias químicas o peligrosas (14).

El manejo inadecuado en su cadena de comercialización y aplicación en cultivos también son causa probable del impacto de las sustancias químicas: la cadena de comercialización no cumple la regulación (15), su sistema de agua va al alcantarillado local, las bodegas comparten otras actividades comerciales, y se encuentran en zonas de actividad comercial regular, el transporte se realiza en vehículos de carga que no cumplen la norma ambiental para transporte de sustancias químicas peligrosas (16), a esto se suman quienes hacen uso de sustancias químicas y usan el transporte público para trasportarlo, la NTC 1692 y la NTC 4702-6.

La ausencia de inspección, vigilancia y control en el cumplimiento normativo y técnico y el uso inadecuado de las sustancias químicas han afectado la salud de todos los grupos poblacionales del Meta; las exposiciones han ocurrido de forma accidental por exposición ambiental, el acceso a estas sustancias es libre, el uso es realizado por personal no capacitado, o por empresas de grandes volúmenes que producen contaminación (del aire, el agua y los alimentos) y exposición ocupacional en campesinos que no utilizan medidas de protección, o trabajadores formales que no cumplen con las normas de protección laboral.

Los resultados obtenidos arrojan que la intoxicación más común en los habitantes de los municipios del Meta es la ocasionada por plaguicidas con el $68 \%$ de la incidencia y el $66 \%$ de la mortalidad, seguida de la intoxicación con otras por otras sustancias con el 27,7\% de los casos de morbilidad y $34 \%$ de la mortalidad. Meta es un departamento caracterizado por su producción agrícola $(17,18)$ y con una frontera agrícola en crecimiento (19), la producción agrícola utiliza plaguicidas para proteger sus productos y mejorar la producción (2023), esto explicaría la ocurrencia mayoritaria de morbilidad y mortalidad atribuible a plaguicidas, los cuales son usados para el control biológico, exponiendo la población a agentes tóxicos, ocasionando enfermedad y muerte por intoxicación $(13,23-25)$. La población del Meta se intoxica mediante tres principales tipos de exposición, primero la accidental, la cual representa el 52,1\% de los casos, seguida de la intencional suicida con $21,2 \%$ y la exposición ocupacional con el 18,1\%. 
El tipo de exposición también se distribuye según el grupo de sustancias químicas de la siguiente manera: las intoxicaciones con intención suicida que ocurren debido al acceso que se tiene de las sustancias y al reconocimiento de las mismas como armas letales $(26,27)$, el $44,9 \%$ de las intoxicaciones con plaguicidas ocurrieron por esta causa, el $24,7 \%$ de las intoxicaciones por otras sustancias obedecieron a intencionalidad suicida.

La exposición ocupacional es mayor con plaguicidas, la cual está ligada a la actividad agrícola y pecuaria (28-30) desarrollada en municipios como Fuente de Oro, San Carlos de Guaroa, El Castillo, Puerto Lleras y El Dorado. El manejo inadecuado de estas sustancias junto con el comportamiento cultural de no reconocer el riesgo inherente al manipular las sustancias aumentan la exposición de los campesinos (12).

La exposición accidental ocurre principalmente con solventes y productos de aseo, este tipo de exposición ocurre por manejo inadecuado de sustancias químicas en la viviendas $(20,27$, 31 ), finalmente la contaminación ambiental contribuye a las exposiciones accidentales, contaminación del aire $(32)$, el agua $(33,34)$ y los alimentos $(35,36)$, además del mal manejo de las sustancias químicas (20).

Las principales vías de exposición a las sustancias químicas en los municipios del Meta fueron: la oral, la respiratoria y la dérmica; el predominio de la vía oral es natural en las exposiciones suicidas y accidentales por el fácil acceso que las personas tienen a la misma, mientras la vía respiratoria y la vía dérmica son reconocidas como las principales vías en la exposición ocupacional $(20,37)$.

Las intoxicaciones ocurren con mayor proporción en hombres y la proporción de menores de edad afectados por las intoxicaciones varía según cada sustancia química; en las intoxicaciones por plaguicidas son el $30 \%$, con otras sustancias $50 \%$, con solventes $70 \%$; el promedio de en la edad es diferente entre los grupos, siendo la intoxicación por solventes la que tiene en promedio de edad más bajo (14,6 años) como afectados, la distribución por edad y sexo es similar la reportada en otros países de Latinoamérica (37).

\section{CONCLUSIONES}

Las actividades humanas en el Meta han traído consigo la utilización de sustancias químicas: solventes, plaguicidas y otras sustancias, esta utilización ocasiona la exposición e intoxicación, generando morbilidad y mortalidad humana. Las intoxicaciones afectan a quienes utilizan los productos en sus actividades laborales, como a personas ajenas a su uso, niños y adultos sin distingo de ocupación ni edad, convirtiendo la intoxicación por sustancia en un problema de salud pública y manejo ambiental para todos los grupos sociales.

El impacto que han ocasionado las sustancias químicas sobre los habitantes de los municipios del Meta evidencian el uso inadecuado de estas sustancias derivado del incumplimiento de la normatividad en el transporte, la comercialización, el almacenamiento, el uso del producto y la disposición final de los residuos, destacando la falta de conocimiento técnico junto con una vigilancia y control poco efectivo por parte de entes de control que han permitido este uso irregular, ocasionando impacto ambiental y en el bienestar,

Se requiere con premura el control de la situación, almacenamiento, uso y disposición final de las sustancias químicas en los municipios del Meta para reducir el impacto de las mismas.

En necesario que la Corporación Autónoma Regional, la Secretaría Departamental de Medio Ambiente, la Secretaría Departamental de Salud y las secretarías homólogas municipales fortalezcan los programas de inspección, vigilancia para un control efectivo en la cadena de uso, transporte y comercialización de las 
sustancia químicas; además, reconocer la afectación del derecho a la vida, el derecho a la salud y a un ambiente sano, derechos vulnerados, por la actividad agrícola, pecuaria y el permanente uso de sustancias en los hogares. Potencial conflicto de intereses: los autores declaran la no existencia de conflicto de intereses en la realización de la investigación.

\section{REFERENCIAS}

1. Moiso A, Barragán H. Determinantes de la salud. Fundamentos de la Salud Pública Argentina. La Plata. Editorial EDULP. 2007.

2. Pérez Jiménez D, Diago Garrido Y, Corona Miranda B, Espinosa Díaz R, González Pérez JE. Enfoque actual de la salud ambiental. Revista Cubana de Higiene y Epidemiología. 2011; 49(1):84-8.

3. Cárdenas O, Silva E, Morales L, Ortiz J. Estudio epidemiológico de exposición a plaguicidas organofosforados y carbamatos en siete departamentos colombianos, 1998-2001. Biomédica. 2005; 25:170-10.

4. Vargas M. La contaminación ambiental como factor determinante de la salud. Revista Española de Salud Pública. 2005; 79:117-11.

5. Gutiérrez $\mathrm{O}$, Martínez $C$. Indicador sintético para la medición de la necesidad de investigación y gestión ambiental basado en morbimortalidad ocurrida en 2009-2012, Meta, Colombia. Luna Azul. 2016; 42:154-13.

6. Vargas M, Pulgarín I. Calidad ambiental interior: bienestar, confort y salud. Rev Esp Salud Pública. 2005; 79(2):243-9.

7. Uribe $M$, Castro R, Paéz I, Carvajal N, Barbosa $E$, León L, et al. Impacto en la salud y el medio ambiente por exposición a plaguicidas e implementación de buenas prácticas agrícolas en el cultivo de tomate, Colombia, 2011. Revista Chilena de Salud Pública. 2012; 16(2):p. 96-11.

8. Conpes 3550. Lineamientos para la formulación de la política integral de salud ambiental con énfasis en los componentes de calidad de aire, calidad de agua y seguridad química, (2008).

9. Instituto Nacional de Salud. Protocolo de vigilancia en salud pública intoxicaciones por sustancias quimicas. 2014. p. 50.

10. Ministerio de Salud y Protección Social. Plan Decenal de Salud Pública 2012-2021: La salud en Colombia la construyes tú. Bogotá D.C. 2013.

11. García JE. Intoxicaciones agudas con plaguicidas: costos humanos y económicos. Revista Panamericana de Salud Pública. 1998; 4(6).

12. Gutiérrez-Strauss AM, Baltazar RG, Estrada GS, Avelar RS, Velasco A, Cortés SL. Veneno para plagas: una aproximación desde la antropología cognitiva sobre exposición laboral, efectos en salud y calidad de vida de los aplicadores de plaguicidas del sector informal rural. Salud Uninorte. 2013; 29(3):501-13.

13. Tabares L, Juan C, López A, Yolanda L. Salud y riesgos ocupacionales por el manejo de plaguicidas en campesinos agricultores, municipio de Marinilla, Antioquia, 2009. Revista Facultad Nacional de Salud Pública. 2011; 29(4):432-13

14. Decreto 2556. Por el cual se adopta la Tabla de Enfermedades Profesionales. (2009).

15. Decreto 1843. Por el cual se reglamentan parcialmente títulos de la ley 09 de 1979 , sobre uso y manejo de plaguicidas. (1991).

16. Castro Ampudia JA, Escarria Díaz J. Identificación de factores de riesgo en el transporte y almacenacimiento de sustancias químicas peligrosas en Propal SA de acuerdo con el decreto 1609 del 2002. [Tesis maestría]. Cali: Universidad Autónoma de Occidente; 2009.

17. Mincomercio. Departamento de Meta. 2013. Disponible en: https://www.mincomercio.gov.co/ publicaciones. php?id=16724.

18. Gutiérrez O. La carretera Bogotá-Villavicencio, su impacto sobre el ordenamiento territorial. Revista Luna Azul. 2015;40:277-16. 
19. Pardo JA, Moreno SH, Jiménez YF. Distribución de la propiedad rural en el departamento del Meta por subregiones. Inquietud Empresarial. 2015; 15(1):189-20.

20. Hernández MM, Jiménez C, Jiménez F, Arceo ME. Caracterización de las intoxicaciones agudas por plaguicidas: perfil ocupacional y conductas de uso de agroquímicos en una zona agrícola del Estado de México, México. Revista Internacional de Contaminación Ambiental. 2007; 23(4):159-9.

21. Ferrer A. Intoxicación por plaguicidas. Anales Sis San Navarra. 2003; 26( Suppl 1 ): 155-171.

22. González-Arias C, Robledo-Marenco M, Medina-Díaz I, Velázquez-Fernández J, Girón-Pérez MI, Quintanilla-Vega b, et al. Patrón de uso y venta de plaguicidas en Nayarit, México. Revista Internacional de Contaminación Ambiental. 2010;26(3):221-8.

23. Karam MÁ, Ramírez G, Montes PB, Galván JM. Plaguicidas y salud de la población. CIENCIA ergo-sum. $2015 ; 11(3): 246-54$.

24. Zamora FR, Torres D, Rodríguez N, Zamora FJ. Impacto del uso de plaguicidas sobre los niveles de colinesterasa total en sangre en productores agrícolas del asentamiento campesino Santa Teresa, Municipio Miranda del Estado Falcón, Venezuela. Revista de la Facultad de Agronomía. 2011;35(2):35 (2): 56-6.

25. González Vides G. Intoxicacion por plaguicidas: casuística del Hospital Universitario del Caribe y de la Clínica Universitaria San Juan de Dios de Cartagena. 2009-2010. [Tesis maestría]: Universidad Nacional de Colombia; 2011.

26. Pérez S, Álvarez M, Baldo D, Capote B. Intoxicaciones agudas por plaguicidas consultadas al Centro Nacional de Toxicología durante el bienio 2007-2008. Revista Cubana de Medicina Militar. 2012; 41(4):415-22.

27. Prado Y, Vizcaíno M, Abeledo CM, Prado E, Leiva O. Intoxicaciones agudas en pediatría. Revista Cubana de Pediatría. 2011; 83(4):356-64.

28. Larrea M, Tirado N, Ascarrunz E. Daño genotóxico por exposición a plaguicidas en agricultores del Municipio de Luribay. Biofarbo. 2010; 18:31-13.

29. Gómez-Pérez R, Rojas G, Miranda-Contreras L, Cruz I, Berrueta L, Salmen S, et al. Efectos de exposición ocupacional a plaguicidas sobre la integridad de la cromatina espermática. Revista Venezolana de Endocrinología y Metabolismo. 2011;9(2):67-13.

30. Yucra S, Gasco M, Rubio J, Gonzales G. Exposición ocupacional a plomo y pesticidas órganofosforados: efecto sobre la salud reproductiva masculina. Revista Peruana de Medicina Experimental y Salud Publica. 2008; 25(4):394-9.

31. Herranz M., Clerigué N. Intoxicación en niños: Metahemoglobinemia. Anales Sis San Navarra . 2003; 26( Suppl 1 ): 209-223.

32. García-Gutiérrez C, Rodríguez-Meza GD. Problemática y riesgo ambiental por el uso de plaguicidas en Sinaloa. Ra Ximhai. 2012; 8(3);1-11

33. Tobón-Marulanda FÁ, López-Giraldo LA, Paniagua-Suárez RE. Contaminación del agua por plaguicidas en un área de Antioquia. Revista de Salud Pública. 2010; 12(2):300-7.

34. Lans E, Marrugo JL, Díaz B. Estudio de la contaminación por pesticidas organoclorados en aguas de la Ciénaga Grande del Valle Bajo del río Sinú. Temas Agrarios. 2008; 13(1);49-8

35. Pérez M, Segura A, García R, Colinas T, Pérez M, Vázquez A, et al. Residuos de plaguicidas organofosforados en cabezuela de brócoli (Brassica oleracea) determinados por cromatografía de gases. Revista Internacional de Contaminación Ambiental. 2009; 25(2):103-10.

36. Murcia A, Stashenko E. Determinación de plaguicidas organofosforados en vegetales producidos en Colombia. Agro sur. 2008; 36(2):71-11.

37. Gutiérrez W, Cerda P, Plaza-Plaza JC, Mieres JJ, Paris E, Ríos JC. Caracterización de las exposiciones a plaguicidas entre los años 2006 y 2013 reportadas al Centro de Información Toxicológica de la Pontificia Universidad Católica de Chile. Revista Médica de Chile. 2015; 143(10):1269-76. 